\title{
A POSSIBLE GRAVITATIONAL LENS IN THE HUBBLE DEEP FIELD SOUTH
}

\author{
Rennan Barkana, Roger Blandford, ${ }^{1}$ and David W. Hogg ${ }^{2}$ \\ Institute for Advanced Study, Olden Lane, Princeton, NJ 08540; barkana@ias.edu \\ Received 1998 December 15; accepted 1999 January 13; published 1999 February 11
}

\begin{abstract}
We model an apparent gravitational lens system HDFS 2232509-603243 in the Hubble Deep Field South. The system consists of a blue $V=25$ mag arc separated by 0.9 from a red $V=22$ mag elliptical galaxy. A mass distribution that follows the observed light distribution with a constant mass-to-light ratio can fit the arc component positions if external shear is added. A good fit is also obtained with simple parameterized models, and all the models predict a fourth image that is fainter than the detection limit. The inferred mass-to-light ratio is $\sim 15$ in solar units if the lens is at $z=0.6$. The lens models predict a velocity dispersion of $\sim 280 \mathrm{~km} \mathrm{~s}^{-1}$ that could be confirmed with spectroscopy.
\end{abstract}

Subject headings: cosmology: observations — galaxies: individual (HDFS 2232509-603243) — gravitational lensing

\section{INTRODUCTION}

Detailed studies of gravitational lenses have provided a wealth of information on galaxies, both through modeling of individual lens systems (see, e.g., Schneider, Ehlers, \& Falco 1992 and Blandford \& Narayan 1992) and from the statistical properties of multiply imaged sources (see, e.g., Turner, Ostriker, \& Gott 1984 and Kochanek 1996). The extraordinary resolution of the Hubble Space Telescope (HST) has allowed for the first time the detection of lenses where both the source and the deflector are "normal," optically selected galaxies. Ratnatunga et al. (1995) discovered two four-image "Einstein cross" gravitational lenses in the Groth-Westphal strip. Their mass models required ellipticities substantially above those of the light distributions, suggesting (as is commonly suspected in radio lenses) that some external shear is important to the lensing.

The Hubble Deep Field (HDF; Williams et al. 1996), the deepest set of exposures taken with the HST, has enabled an unprecedented study of galaxy morphological evolution (see, e.g., Ellis 1998) and global star formation history (see, e.g., Madau, Pozzeti, \& Dickinson 1998). Candidate gravitational lenses were reported by Hogg et al. (1996), who noted, on the basis of radio surveys, that several lenses are expected in the HDF (cf. Cooray, Quashnock, \& Miller 1999). However, after further observations, not one strong case for multiple imaging has been identified (Zepf, Moustakas, \& Davis 1997; J. Cohen 1998, private communication).

The southern Hubble Deep Field (HDF-S; Williams et al. 1998) is a second set of ultradeep HST exposures, and several lenses are again expected. The best candidate lens, noted by the HDF-S team (R. Williams 1998, private communication), consists of a blue arc about 0.9 from an elliptical galaxy. In this Letter, we report on a detailed study of this arc and argue that it is a bona fide multiply imaged source.

\section{OBSERVATIONS}

The Wide Field Planetary Camera 2 (WFPC2) HDF-S is a $\sim 5 \operatorname{arcmin}^{2}$ field centered on $22^{\mathrm{h}} 32^{\mathrm{m}} 56^{\mathrm{s}} .22,-60^{\circ} 33^{\prime} 02^{\prime \prime} .69$ (J2000), imaged with the WFPC2 instrument on the HST in

\footnotetext{
${ }^{1}$ Also California Institute of Technology, Pasadena, CA 91125.

${ }^{2}$ Hubble Fellow.
}

the F300W, F450W, F606W, and F814W bandpasses for total exposure times of $1.4 \times 10^{5}, 1.0 \times 10^{5}, 8 \times 10^{4}$, and $1.0 \times$ $10^{5} \mathrm{~s}$, respectively, reaching point-source sensitivities of roughly $V=29$ mag (Williams et al. 1998). For the "version 1.0 " reductions of the images, we adopt the Vega-relative calibrations supplied by the HDF-S team whereby 1 DN corresponds to $\mathrm{F} 300 \mathrm{~W}=19.43 \mathrm{mag}, \mathrm{F} 450 \mathrm{~W}=22.02 \mathrm{mag}$, $\mathrm{F} 606 \mathrm{~W}=22.90 \mathrm{mag}$, or $\mathrm{F} 814 \mathrm{~W}=21.66 \mathrm{mag}$. The images clearly show a bright candidate lens system at pixel location (2982.7, 2837.6). With the pixel scale of 0.0398 , this is also $22^{\mathrm{h}} 32^{\mathrm{m}} 50^{\mathrm{s}} 90,-60^{\circ} 32^{\prime} 43^{\prime \prime} .0$ (J2000). This candidate lens system will be called "HDFS 2232509-603243" hereafter.

The morphology of HDFS 2232509-603243 is that of a $V \sim 22$ mag elliptical galaxy (hereafter "the elliptical") with a much bluer arc (hereafter "the arc") located 0 " 9 to the northwest from the center of the elliptical, as shown in Figure 1. There is also a faint pointlike source (hereafter "the dot") located 0 ".96 to the northeast from the center of the elliptical.

The elliptical is smooth and symmetrical, so images created by rotating its images through $180^{\circ}$ (centered on the pixel nearest the center of the elliptical) and subtracting them from their unrotated originals show essentially no sign of the elliptical. Photometry of the arc was performed in these "rotatedsubtracted" images. The total F606W flux (given in Table 1) was measured through a partial annulus of inner radius 0 ".517 and outer radius 1 ".194, extending over position angles $0^{\circ}$ (north through east) through $-108^{\circ}$. Colors were measured through a partial annulus of inner and outer radii 0.677 and $1^{\prime \prime} 035$, respectively, over $-18^{\circ}$ through $-90^{\circ}$. The flux and colors of the dot were measured through a 0.159 radius circular aperture. Because nearby pixels are correlated, signal-to-noise ratios for photometric measurements were found by rebinning the image into pixels $4 \times 4$ times larger.

The arc separates into four distinct components, labeled A, $\mathrm{B}, \mathrm{C}$, and $\mathrm{D}$, going clockwise around the arc. All have very similar colors. Components A, B, and D appear to have pointlike centers with fuzz, while component $\mathrm{C}$ is more extended. Gaussians with the same FWHM as the point-spread function (4 pixels) were fitted to components $\mathrm{A}, \mathrm{B}$, and $\mathrm{D}$, and a Gaussian of variable width was fitted to component $\mathrm{C}$, all in the F450W rotated-subtracted image. The best-fit positions and fluxes of these Gaussians are given in Table 2.

In order to make arc-free images of the elliptical, the region 


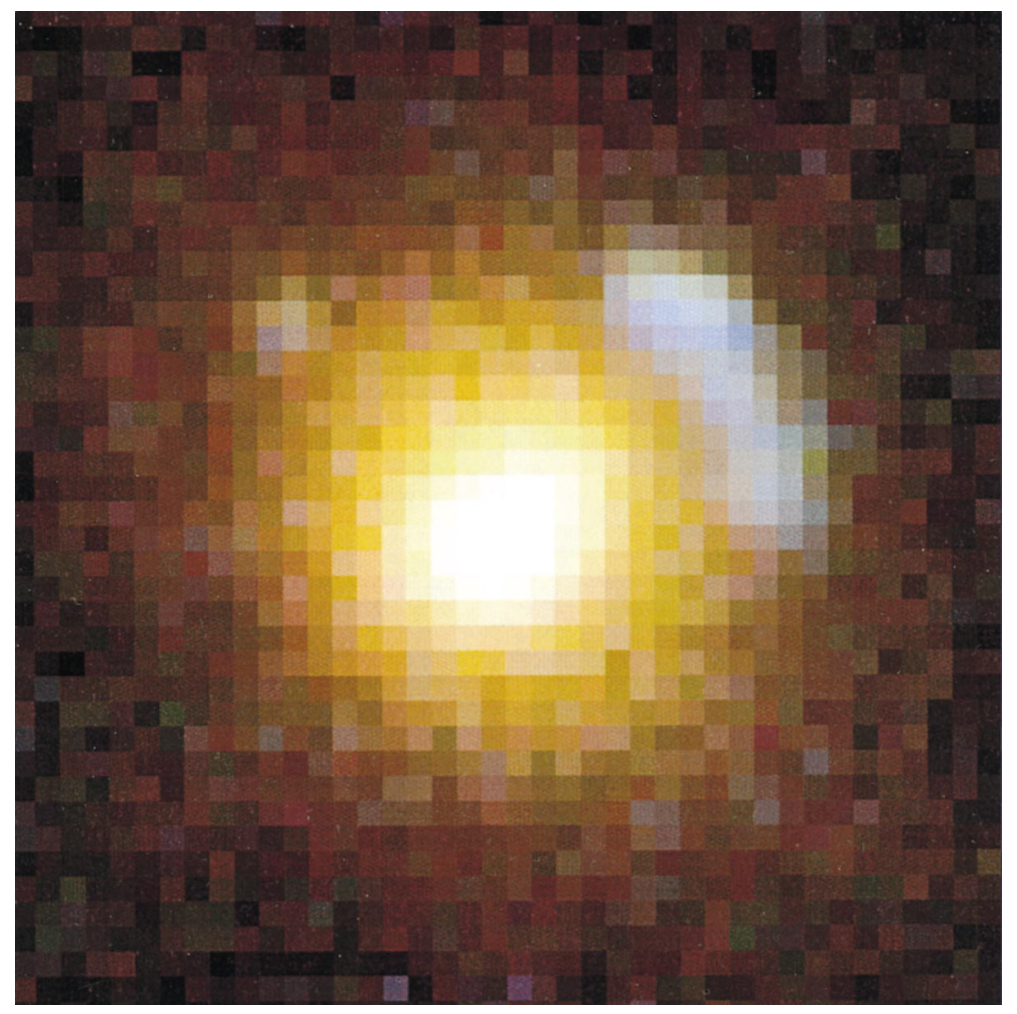

FIG. 1.-A region taken from the public release of the HDF-S (Williams et al. 1998). It shows the lens galaxy, the arc to its northwest, and the dot to its northeast. North is up, and east is to the left (except for a 0.5 rotation). The figure is centered on the lens galaxy and measures 3.2 on a side.

of each image in the partial annulus centered on the elliptical with inner and outer radii of 0.517 and 1".194, respectively, and position angles $0^{\circ}$ through $-108^{\circ}$ was replaced with the corresponding region diametrically opposite (i.e., at a position angle rotated by $180^{\circ}$ ). These "arc-removed" images show virtually no evidence of the arc. The total F814W flux of the elliptical was measured out to its $2 \sigma$ (per pixel) isophote. The colors were measured through 0 ".915 radius circular apertures and are given in Table 1.

A de Vaucouleurs profile is a very good fit to the azimuthally averaged radial profile of the elliptical in the arc-removed images. The effective radius is $r_{e}=0.31$, and the F814W-band surface brightness at the effective radius is $21.0 \mathrm{mag}$ in each $\operatorname{arcsec}^{2}$. The isophote ellipticity orientation varies with radius. The outer isophotes (radii $>0$ ".8) of the elliptical have an ellipticity of $\sim 0.2$ and are elongated along $40^{\circ}$. The inner isophotes (radii $<0$ ".3) also have an ellipticity of $\sim 0.2$ but are elongated along $-40^{\circ}$, i.e., almost orthogonal. Isophotes intermediate to these two regimes are fairly circular.

A comparison of the colors of the elliptical with HDF galaxies with known redshifts suggests that its redshift is roughly $z=0.6$ (cf. the photometric redshift of 0.5 of Gwyn 1998). This suggests a total luminosity ${ }^{3}$ of $8.7 \times 10^{9} h^{-2} L_{\odot}$ in the rest-frame $B$ band, or roughly $L_{*}$. The luminosity is a factor of 3 larger if $z=0.8$ and a factor of 6.5 smaller if $z=0.3$.

\section{LENS MODELS}

We consider several models for the lensing mass distribution, simple parameterized models as well as a constant mass-tolight ratio model. The image positions are not very sensitive to the radial profile of the lens, and so, for the parameterized models, we take it to be singular and isothermal. Asymmetry is required, and we consider two types: external shear or an elliptical galaxy. We assume that the arc components A, B, and $\mathrm{D}$ are three images of a common source, with $\mathrm{A}$ and $\mathrm{B}$ constituting a merging pair. Component $\mathrm{C}$ can easily be produced if the source is extended or if it is a multicomponent. We do not include as constraints component $\mathrm{C}$ or the observed flux

\footnotetext{
${ }^{3}$ We assume a world model of $\Omega=0.3$ in matter and no cosmological constant, and we also set $H_{0}=100 h \mathrm{~km} \mathrm{~s}^{-1} \mathrm{Mpc}^{-1}$.
}

TABLE 1

PHOTOMETRY

\begin{tabular}{|c|c|c|c|c|c|c|c|c|}
\hline \multirow[b]{2}{*}{ COMPONENT } & \multicolumn{2}{|c|}{ FLUX $^{\mathrm{a}}$} & \multicolumn{2}{|c|}{ F300W-F450W } & \multicolumn{2}{|c|}{ F450W-F606W } & \multicolumn{2}{|c|}{ F606W-F814W } \\
\hline & (mag) & $\mathrm{S} / \mathrm{N}^{\mathrm{b}}$ & (mag) & $\mathrm{S} / \mathrm{N} 1, \mathrm{~S} / \mathrm{N} 2^{\mathrm{c}}$ & (mag) & $\mathrm{S} / \mathrm{N} 1, \mathrm{~S} / \mathrm{N} 2$ & (mag) & $\mathrm{S} / \mathrm{N} 1, \mathrm{~S} / \mathrm{N} 2$ \\
\hline Elliptical & $\mathrm{F} 814 \mathrm{~W}=20.52$ & 950 & 0.31 & $9.2,99$ & 1.86 & 99,610 & 1.53 & 610,1200 \\
\hline $\operatorname{Arc} \ldots \ldots \ldots \ldots$ & $\mathrm{F} 606 \mathrm{~W}=25.19$ & 41 & -0.96 & 11,37 & 0.24 & 37,51 & 0.40 & 51,37 \\
\hline Dot $\ldots \ldots \ldots \ldots \ldots$ & $\mathrm{F} 606 \mathrm{~W}=28.70$ & 6.2 & $\ldots$ & $-1.5,3.9$ & 0.39 & $3.9,6.2$ & -0.05 & $6.2,2.9$ \\
\hline
\end{tabular}

\footnotetext{
${ }^{a}$ Different components have flux measurements in different bands and through different focal-plane apertures (see text for details).

${ }^{\mathrm{b}}$ Signal-to-noise ratios (S/Ns) measure the flux through the aperture relative to the expected sky noise rms through that same aperture (see text for aperture definitions).

${ }^{\mathrm{c}}$ The $\mathrm{S} / \mathrm{N}$ values $\mathrm{S} / \mathrm{N} 1$ and $\mathrm{S} / \mathrm{N} 2$ are for the two bandpasses involved in the color measurement, with bluer first.
} 
TABLE 2

ASTROMETRY $^{\mathrm{a}}$ AND PHOTOMETRY

\begin{tabular}{|c|c|c|c|}
\hline Subcomponent & $\begin{array}{c}\Delta \alpha \\
(\operatorname{arcsec})\end{array}$ & $\begin{array}{c}\Delta \delta \\
(\operatorname{arcsec})\end{array}$ & $\begin{array}{c}\mathrm{F} 450 W^{\mathrm{b}} \\
(\mathrm{mag})\end{array}$ \\
\hline Elliptical & 0 & 0 & \\
\hline $\operatorname{Arc} \mathrm{A} \ldots$ & 0.406 & 0.777 & 27.55 \\
\hline Arc $B \ldots \ldots \ldots$ & 0.568 & 0.721 & 27.43 \\
\hline Arc C & 0.711 & 0.478 & 27.08 \\
\hline Arc $D \ldots \ldots \ldots$ & 0.824 & 0.192 & 28.10 \\
\hline Dot $\ldots \ldots \ldots \ldots$ & -0.778 & 0.567 & $\cdots$ \\
\hline
\end{tabular}

${ }^{\mathrm{a}}$ Positions are given relative to $22^{\mathrm{h}} 32^{\mathrm{m}} 50^{\mathrm{s}} 9017$, $-60^{\circ} 32^{\prime} 43^{\prime \prime} .009$ (J2000), with the $\alpha$-direction west and the $\delta$-direction north. We estimate position errors of 0.02 from fitting an artificial image.

${ }^{b}$ The magnitudes of the arc components are based on the fluxes of the fitted Gaussians. The fluxes do not add up to the total arc flux because the magnitudes exclude some diffuse emission.

ratios among components $\mathrm{A}, \mathrm{B}$, and $\mathrm{D}$, since these are sensitive to the detailed flux distribution of the source. We discuss the dot below.

We consider a lens at redshift $z_{L}$ and a source at $z_{S}$. Then the lens mass distribution determines a convergence $\kappa$ and a potential $\psi$ (see, e.g., Schneider et al. 1992 and Blandford \& Narayan 1992). The singular isothermal sphere (SIS) is defined by $\kappa=b /(2 r)$. We add to the potential the term $\psi_{\gamma}=$ $-\frac{1}{2} \gamma r^{2} \cos 2\left(\theta-\theta_{\gamma}\right)$, which defines an external shear of magnitude $\gamma$ and direction $\theta_{\gamma}$. If the shear is due to an axisymmetric galaxy, then it is located at an angle $\theta_{\gamma}$ from the lens galaxy. By SIS $+\gamma$, we denote the SIS model with external shear.

A singular isothermal elliptical mass distribution (SIEMD) with an axis ratio $a$ and a major axis along the $y$-axis is given by $\kappa=b /\left[2\left(x^{2}+y^{2} a^{2}\right)^{1 / 2}\right]$. More generally, we rotate the major axis by an angle $\theta_{\epsilon}$. The lensing properties of the SIEMD have been studied by Kassiola \& Kovner (1993) and by Kormann, Schneider, \& Bartelmann (1994).

We fitted these two lens models, the SIS $+\gamma$ model and the SIEMD, to the three observed image positions, with the results shown in Table 3. Both lens models have an elongated diamond caustic with four cusps. We indicate a source near a cusp on the major axis by the subscript "maj" and one near a minor cusp by the subscript "min." Since the observed position B is farther from the center of the lens than A or D, the arc is more naturally produced by a minor cusp for which only the middle image of the three is outside the critical curve.

Interestingly, the orientation of the $\mathrm{SIEMD}_{\text {min }}$ model agrees with the observed orientation of the elliptical's outer isophotes, while the orientation of the SIEMD maj $_{\text {model agrees with that }}$ of the inner isophotes. However, among the simple parameterized models, only the SIS $+\gamma_{\text {min }}$ model fits the data well (see Table 3 and Fig. 2). This model requires a substantial shear

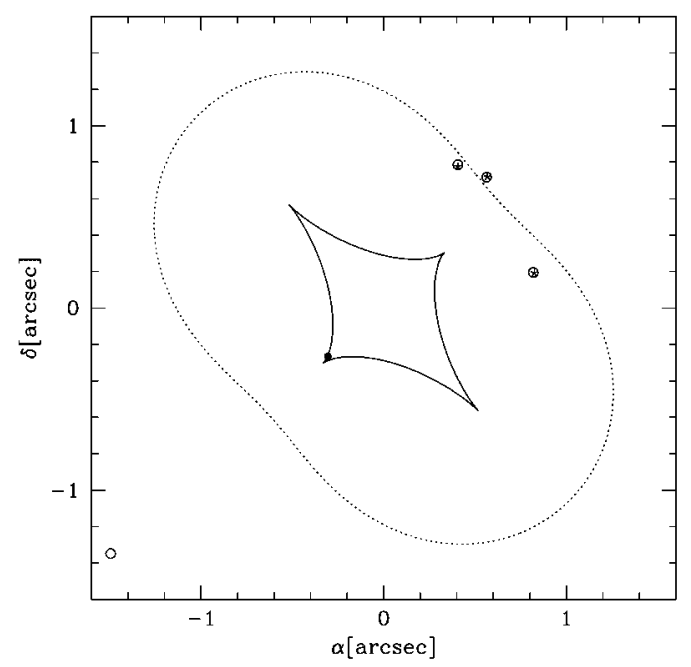

FIG. 2.- Image and source positions for the SIS $+\gamma_{\min }$ model (fourth column of Table 3). Four image positions (open circles) are indicated, along with the source position (filled circle) and the observed positions of arc components $A, B$, and $D$ (asterisks). The tangential caustic (solid) and critical (dotted) curves are also shown. The figure is centered on the lens galaxy, with the $\alpha$ direction west and the $\delta$-direction north.

$(\gamma=0.26)$ that is produced by objects lying in the northeast or southwest direction. To compute the mass-to-light ratio for various models, we fix a circle about the galaxy center of radius 0.915 . The luminosity of the galaxy within this circle is $7.6 \times 10^{9} h^{-2} L_{\odot}$ if $z_{L}=0.6$. If, for example, $z_{S}=1.5$, then the mass within the same circle of the SIS is $M=2.1 \times$ $10^{11} h^{-1}\left(b / 1^{\prime \prime}\right) M_{\odot}$. This yields a mass-to-light ratio of 27 $h\left(b / 1^{\prime \prime}\right)$ and a velocity dispersion $\sigma=270\left(b / 1^{\prime \prime}\right)^{1 / 2} \mathrm{~km} \mathrm{~s}^{-1}$.

As a second approach to modeling, we utilize the resolved image of the lens galaxy (smoothed with a 4 pixel FWHM Gaussian) to construct a model with a constant mass-to-light ratio (denoted $M / L$ ). The pure $M / L$ model fails, but, together with the external shear, it produces an excellent fit, albeit with a large shear. Even a small shear such as 0.05 produces a substantially better fit than a model with no shear (see Table 3 and Fig. 3). All the $M / L$ models produce a minor cusp configuration. The parameter $\bar{\kappa}$ for the $M / L$ models gives the mean $\kappa$ in a circle of radius 0.915 about the galaxy center. If $z_{L}=$ 0.6 and $z_{S}=1.5$, then the mass within this circle is $M=$ $1.9 \times 10^{11} h^{-1} \bar{\kappa} M_{\odot}$, which yields a mass-to-light ratio of $25 h \bar{\kappa}$. If we assume a small shear, then $\bar{\kappa} \sim 1$ (Table 3), and the mass-to-light ratio is on the high side of the range of other lens galaxies (Keeton, Kochanek, \& Falco 1998). Note, however, that we only have an estimated photometric redshift for the lens and a guess for the source redshift. The mass-to-light

TABLE 3

Best-Fit Model Results

\begin{tabular}{|c|c|c|c|c|c|c|c|}
\hline Parameter & SIEMD $_{\text {min }}$ & SIEMD $_{\text {maj }}$ & $\mathrm{SIS}+\gamma_{\min }$ & $\mathrm{SIS}+\gamma_{\text {maj }}$ & $M / L$ & $M / L+\gamma$ & $\begin{array}{c}M / L+\gamma \\
\text { (with } \gamma=0.05 \text { ) }\end{array}$ \\
\hline$b$ or $\bar{\kappa}$ & 0.85 & 0.84 & 1.08 & 0.87 & 1.10 & 1.40 & 0.998 \\
\hline$a$ or $\gamma \ldots$ & $0.03_{-0.03}^{+0.97}$ & $0.96_{-0.74}^{+0.04}$ & $0.26_{-0.05}^{+0.04}$ & $0.005_{-0.005}^{+0.18}$ & $\ldots$ & $0.52_{-0.14}^{+0.15}$ & 0.05 \\
\hline$\theta_{\epsilon}$ or $\theta_{\gamma} \ldots$ & $41.2_{-2.6}^{+2.9}$ & $-48^{\circ} 6_{-3.1}^{+3.0}$ & $42^{\circ} .5_{-0.7}^{+0.7}$ & $-49^{\circ} 0_{-2.8}^{+3.5}$ & $\ldots$ & $43.5_{-0.5}^{+0.5}$ & 53.4 \\
\hline Degrees of freedom ....... & 1 & 1 & 1 & 1 & 3 & 1 & 1 \\
\hline $\bar{\chi}^{2} \ldots \ldots \ldots \ldots \ldots \ldots \ldots$ & 5.1 & 5.6 & 0.26 & 5.8 & 97 & 0.40 & 4.5 \\
\hline
\end{tabular}

Note. - The best fits are formally at $a \rightarrow 0$ for the SIEMD min $_{\text {model, at } a \rightarrow 1 \text { for the SIEMD }}$ model, and at $\gamma \rightarrow 0$ for the SIS $+\gamma_{\text {maj }}$ model. The overall best fit for the $M / L+\gamma$ model is given in the seventh column, while the best fit for this model with the restriction $\gamma=0.05$ is given in the eighth column. Where given, parameter ranges indicate $1 \sigma$ uncertainties defined by $\Delta \chi^{2}=\bar{\chi}^{2}$. All angles are measured north through east. 


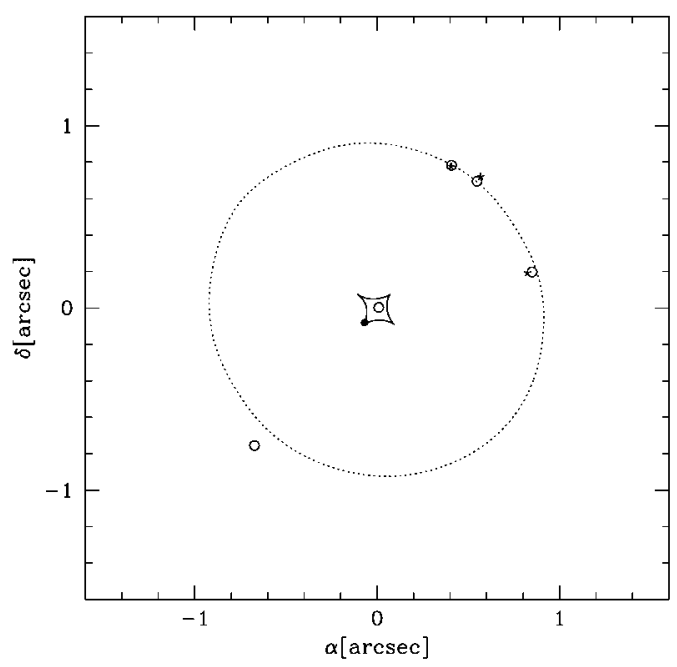

FIG. 3.-Same as Fig. 2, but for the $M / L+\gamma$ model with $\gamma=0.05$ (eighth column of Table 3 ). There are five images, although the one near the lens center is extremely faint.

ratio changes to $14 h \bar{\kappa}$ for $\left(z_{L}=0.8, z_{S}=2.0\right)$ and to $57 h \bar{\kappa}$ for $\left(z_{L}=0.3, z_{S}=1.0\right)$.

No fourth image is detected down to the detection threshold of about 28.9 mag in F606W. This is consistent with the models, which predict a fourth image that is fainter than the third by a factor of 3 or 4 . The $M / L$ models are nonsingular and predict a fifth image that is fainter by an additional factor of $\sim 100$. The best-fitting models also locate the image of the cusp near the observed position of component $\mathrm{C}$, and so this component can be produced if the source is extended.

Thus far, we have not included the dot, assuming it to be a separate background source. We also consider the possibility that the dot is the third image corresponding to components $\mathrm{A}$ and B. The types of lens models used above can fit these alternative image positions with similar $\chi^{2}$ values as in Table 3 , but they fail because they predict an observable arc that includes counterimages of components $\mathrm{C}$ and $\mathrm{D}$ and that extends southeast from the dot. More elaborate lens models could work, but only if the sources of components $\mathrm{C}$ and $\mathrm{D}$ are small (subpixel) and lie right on the caustic.

\section{DISCUSSION}

We have modeled a candidate gravitational lens, HDFS 2232509-603243, that was found in the HDF-S. The image positions can be fitted in a minor cusp configuration by simple lens models that include ellipticity or shear, or with shear added to a model with a constant mass-to-light ratio. However, an exact fit is obtained only with a substantial shear that is produced by objects lying to the northeast or southwest. No obvious candidate galaxy lies within $5^{\prime \prime}$ of the lens, but there are several galaxies $\sim 20^{\prime \prime}$ to the southwest and others $\sim 35^{\prime \prime}$ to the northeast with photometric redshifts in the range of $0.5-0.7$ (Gwyn 1998). Spectroscopic redshifts will determine the possible presence of galaxy groups.

The lensing galaxy is expected to have a redshift of $z \sim$ 0.6 , which gives it a luminosity of $\sim L^{*}$. Lens models imply a mass-to-light ratio of $\sim 25 h$ and a velocity dispersion of $\sim 280$ $\mathrm{km} \mathrm{s}^{-1}$. If the elliptical falls on the fundamental plane, a central line-of-sight velocity dispersion of only $\sim 100 \mathrm{~km} \mathrm{~s}^{-1}$ is expected if $z=0.6$ (Jorgensen, Franx, \& Kjærgaard 1996), which suggests that $z \sim 0.8$ is more likely.

Showing that the arc and the lens galaxy are at different redshifts would rule out the possibility that the arc is a tidally disrupted satellite. On $10 \mathrm{~m}$-class telescopes, it is straightforward to get spectroscopic redshifts of galaxies at $22 \mathrm{mag}$, even elliptical galaxies, which tend to have absorption feature-dominated spectra (Cohen et al. 1999). Some elliptical galaxies at this flux level even have measured central velocity dispersions (van Dokkum et al. 1998; Pahre 1998). Thus, the prospects for follow-up spectroscopy on the elliptical are good. It is common to obtain redshifts for sources at $25 \mathrm{mag}$, even some that are close to bright nearby sources (Steidel et al. 1996). A redshift for the arc is likely obtainable if it is at $z<1.3$ since its blue color suggests that it will show strong emission lines in a visual spectrum (Hogg et al. 1998). The arc is unlikely to be at redshift $z>2.3$ since it shows significant F300W flux.

It is a pleasure to thank Bob Williams and the entire HDF$\mathrm{S}$ team for taking, reducing, and making public the beautiful images of the HDF-S. Scott Burles, Wayne Hu, and Inger Joergensen provided very useful help in short order. We thank the referee, Emilio Falco, for useful comments. This research is based on observations made with the NASA/ESA Hubble Space Telescope, which is operated by AURA, Inc., under NASA contract NAS5-26555. R. Barkana acknowledges support from Institute Funds. R. Blandford acknowledges the Alfred P. Sloan Foundation. D. W. Hogg acknowledges a Hubble Fellowship grant HF-01093.01-97A from STScI, which is operated by AURA, Inc., under NASA contract NAS5-26555.

\section{REFERENCES}

Blandford, R. D., \& Narayan, R. 1992, ARA\&A, 30, 311

Cohen, J. G., Hogg, D. W., Pahre, M. A., Blandford, R., Shopbell, P. L., \& Richberg, K. 1999, ApJS, in press

Cooray, A. R., Quashnock, J. M., \& Miller, M. C. 1999, ApJ, 511, 562

Ellis, R. S. 1998, Nature, 395, A3

Gwyn, S. 1998, in preparation

Hogg, D. W., Blandford, R. D., Kundić, T., Fassnacht, C. D., \& Malhotra, S. 1996, ApJ, 467, L73

Hogg, D. W., Cohen, J. G., Blandford, R., \& Pahre, M. A. 1998, ApJ, 504, 622

Jorgensen, I., Franx, M., \& Kjærgaard, P. 1996, MNRAS, 280, 167

Kassiola, A., \& Kovner, I. 1993, ApJ, 417, 450

Keeton, C. R., Kochanek, C. S., \& Falco, E. E. 1998, ApJ, 509, 561

Kochanek, C. S. 1996, ApJ, 466, 638

Kormann, R., Schneider, P., \& Bartelmann, M. 1994, A\&A, 284, 285
Madau, P., Pozzeti, L., \& Dickinson, M. 1998, ApJ, 498, 106

Pahre, M. A. 1998, ApJS, submitted

Ratnatunga, K. U., Ostrander, E. J., Griffiths, R. E., \& Im, M. 1995, ApJ, 453, L5

Schneider, P., Ehlers, J., \& Falco, E. E. 1992, Gravitational Lenses (New York: Springer)

Steidel, C. C., Giavalisco, M., Dickinson, M., \& Adelberger, K. L. 1996, AJ, 112,352

Turner, E. L., Ostriker, J. P., \& Gott, J. R. 1984, ApJ, 284, 1

van Dokkum, P. G., Franx, M., Kelson, D. D., \& Illingworth, G. D. 1998, ApJ, 504, L17

Williams, R., et al. 1996, AJ, 112, 1335

- 1998, in preparation

Zepf, S. E., Moustakas, L. A., \& Davis, M. 1997, ApJ, 474, L1 